\title{
ON FAST MAGNETIC FIELD RECONNECTION
}

\author{
E. R. PRIEST \\ Dept. of Applied Mathematics, The University, St. Andrews, Scotland \\ and \\ A. M. SOWARD \\ School of Mathematics. The University, Newcastle-upon-Tyne, England
}

\begin{abstract}
The first model for 'fast' magnetic field reconnection at speeds comparable with the Alfven speed was put forward by Petschek (1964). It involves one shock wave in each quadrant radiating from a central diffusion region and leads to a maximum reconnection rate dependent on the electrical conductivity but typically of order $10^{-1}$ or $10^{-2}$ of the Alfvén speed. Sonnerup (1970) and Yeh and Axford (1970) then looked for similarity solutions of the magnetohydrodynamic equations, valid at large distances from the diffusion region; by contrast with Petschek's analysis, their models have two waves in each quadrant and produce no sub-Alfvénic limit on the reconnection rate.

Our approach has been, like Yeh and Axford, to look for solutions valid far from the diffusion region, but we allow only one wave in each quadrant, since the second is externally generated and so unphysical for astrophysical applications. The result is a model which qualitatively supports Petschek's picture; in fact it can be regarded as putting Petschek's model on a firm mathematical basis. The differences are that the shock waves are curved rather than straight and the maximum reconnection rate is typically a half of what Petschek gave. The paper is a summary of a much larger one (Soward and Priest, 1976).
\end{abstract}

\section{Introduction}

The basic principles for two-dimensional magnetic field reconnection in a highly conducting plasma were established many years ago by Dungey (1953), Sweet (1958) and Parker (1963). They consider oppositely-directed magnetic field lines which are carried towards one another by a converging plasma flow, as shown in Figure 1. The magnetic field is assumed frozen to the plasma, save in a 'diffusion' region of dimensions $l$ and $L$ (and $L$ out of the plane of the figure). There the electric current density is so large that the field lines can slip through the plasma. They enter the diffusion region at a speed $v_{i}$, are reconnected at the neutral point $N$, where the magnetic field strength vanishes, and are carried out of the region with speed $v_{0}$. The outflow magnetic field strength $B_{0}$ is less than the inflow strength $B_{i}$, so that some of the magnetic flux is annihilated in the diffusion region. The corresponding fall in magnetic energy appears partly as heat, through ohmic dissipation, and partly as an increase in the kinetic energy of the plasma.

For steady, incompressible flow Sweet and Parker derived the following order of magnitude relationships between the input and output parameters:

$$
\begin{aligned}
v_{0} & =v_{\mathrm{A}} \equiv B_{i} /(\mu \rho)^{1 / 2}, \\
v_{i} & =\eta / l, \\
v_{i} L & =v_{0} l,
\end{aligned}
$$

where $\eta=\left(\mu_{0} \sigma\right)^{-1}$ is the magnetic diffusivity and $\sigma$ the electrical conductivity. The first arises because the plasma is accelerated away from $N$ by the magnetic tension 


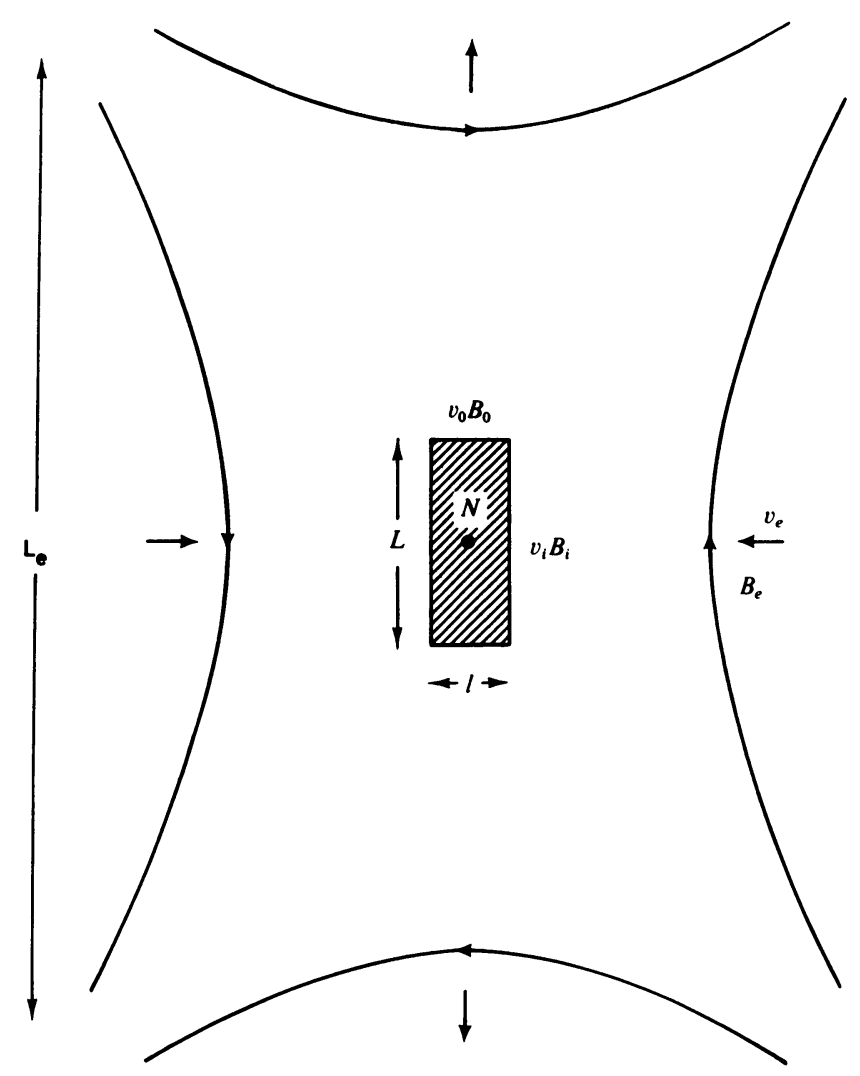

Fig. 1. The overall magnetic configuration for the reconnection process. Oppositely directed magnetic fields of strength $B_{e}$ are carried towards a magnetic neutral point with speed $v_{e}$ from an 'external region' whose scale length is $L_{e}$. Surrounding $N$ is a 'diffusion region' with dimensions $l$ and $L$ where the electric current density is strong. Subscripts $i$ and $o$ refer to inflow and outflow values at the edge of the diffusion region.

$B_{i}^{2} / \mu$ and an excess gas pressure of the same order. The second expresses the fact that flux is being carried in at the speed with which it diffuses through the plasma. The final equation results from the principle of mass conservation.

For given input values $v_{i}, B_{i}$, (i)-(iii) determine $v_{0}$ and the dimensions of the diffusion region. In particular one finds

$$
L=v_{\mathrm{A}} \eta / v_{i}^{2}
$$

which must be compared with a typical overall dimension $L_{e}$ for variations in the magnetic field. If

$$
v_{i} \gg\left(v_{\mathrm{A}} \eta / L_{e}\right)^{1 / 2},
$$

then $L$ is much less than $L_{e}$ in value and the diffusion region occupies only a small part of the flow. Recent attention has been concentrated on such a situation, which we refer to as 'fast' reconnection and which is relevant for most astrophysical and geophysical applications. In particular, the object has been to study the 'external' 
region which surrounds the diffusion region and to determine the maximum allowable value of the speed $v_{e}$ with which magnetic flux (of strength $B_{e}$ at a distance $L_{e}$ ) can be carried towards the neutral point and reconnected. It is necessary to distinguish between the flow speeds $v_{e}$ and $v_{i}$, since, as the plasma approaches the diffusion region it may well be greatly accelerated.

In the next section we summarise critically the present fast reconnection models of the external region; the plasma is assumed perfectly conducting so the diffusion region is regarded as a point at the origin or a line through it. In Section 3 a brief account is given of an asymptotic similarity treatment which we have recently completed (Soward and Priest, 1976).

\section{Previous Models of Fast Reconnection}

\subsection{Petschek}

The first to set up a qualitative mechanism for fast reconnection was Petschek (1964). He noted that, as plasma crosses a slow magnetohydrodynamic shock wave (a finite Alfvén wave in the incompressible limit), the magnetic field direction rotates towards the normal. This fact enabled him to construct a model of the external region with one shock in each quadrant of the $x, y$ plane. The first quadrant is shown in Figure 2a, and the configuration in the rest of the $x, y$ plane can be constructed from it by assuming symmetry about both the $x$ - and $y$-axes. The wave remains stationary at the position OA while plasma and magnetic flux passes through it. In the process, magnetic energy is converted into kinetic energy and heat. Both the wave and the field lines which have passed through it are assumed to be straight.

Petschek estimates the magnetic field strength to the right of the diffusion region (considered as a straight line rather than a point) by assuming OA to coincide with the vertical axis. He then uses Equations (i)-(iii) to link the external region to the diffusion region and gives a qualitative argument for expecting the inflow speed $v_{e}$ to possess a maximum value. The maximum depends weakly on the magnetic Reynolds number $R_{m e}=v_{\mathrm{A} e} L_{e} / \eta$ and is typically 0.1 or 0.01 times the Alfvén speed $v_{\mathrm{A} e}=$ $B_{e} /(\mu \rho)^{1 / 2}$. In view of the qualitative nature of Petschek's estimate, Roberts and Priest (1975) recently attempted to estimate the maximum inflow speed or, loosely speaking, 'reconnection rate' more quantitatively. They solve for the magnetic field in the region to the right of $O A$, assuming $O A$ to be inclined at an angle $\alpha$ to the vertical. After matching to the diffusion region, they plot the reconnection rate $v_{e}$ as a function of $\alpha$ and find that $v_{e}$ does indeed possess a maximum value. Typical ones are $0.1 v_{\mathrm{Ae}}$ for $R_{m e}=10^{2}$ and $0.02 v_{\mathrm{Ae}}$ for $R_{m e}=10^{6}$.

But Petschek's mechanism was not generally accepted. For instance, Sweet and Green (1966) and Priest (1972) showed that, if the shock $O A$ is straight, the field lines to the left of $O A$ are bowed away from $O$, rather than being straight as indicated in Figure 2a. (However, Vasyliunas (1975) has recently claimed that the resulting transition from convex to concave field lines as the plasma leaves the diffusion region presents no problem.) Even more doubt on the validity of Petschek's analysis was cast by the appearance of the alternative solutions due to Sonnerup (1970) and Yeh and Axford (1970). 

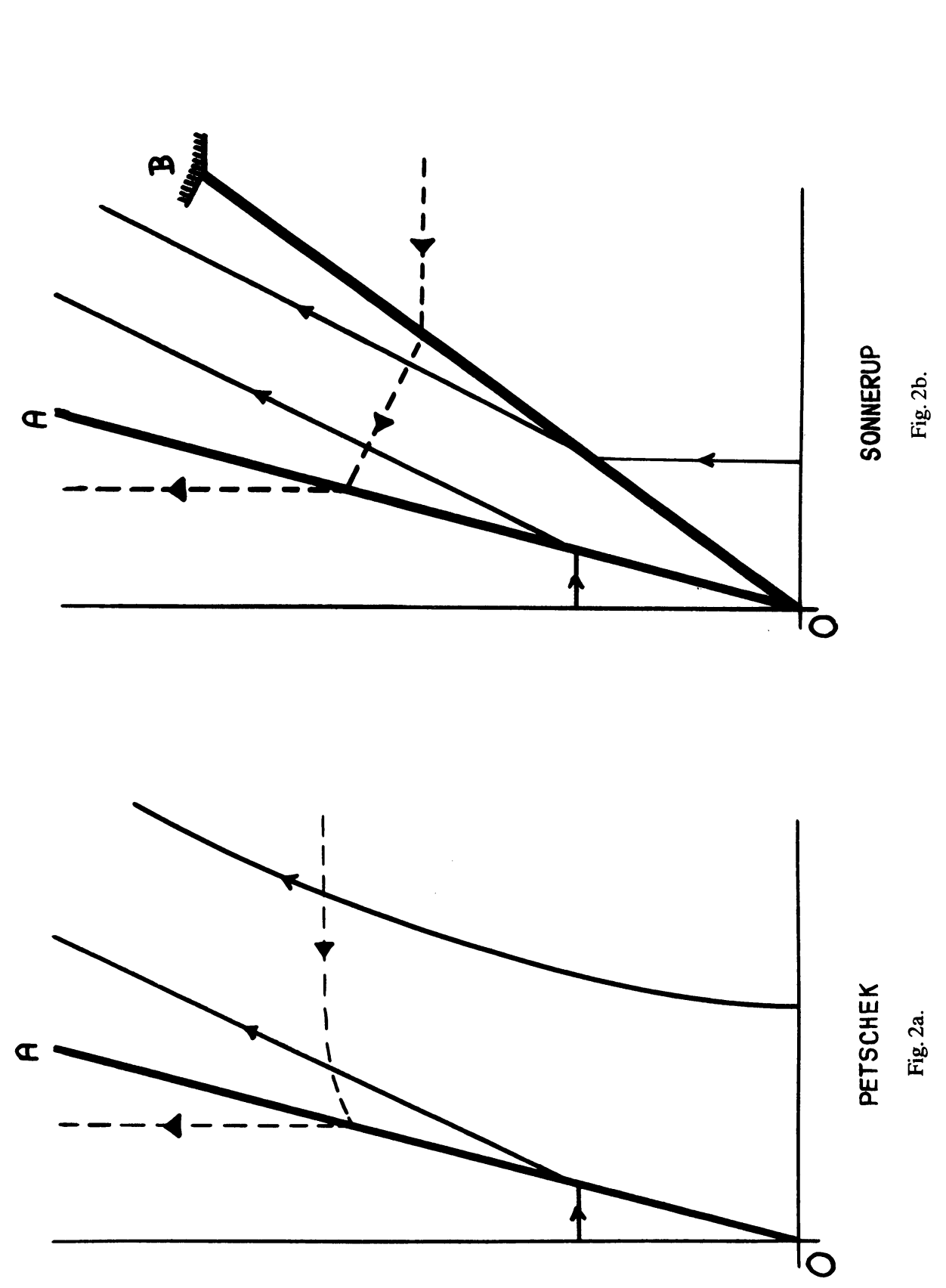

() 


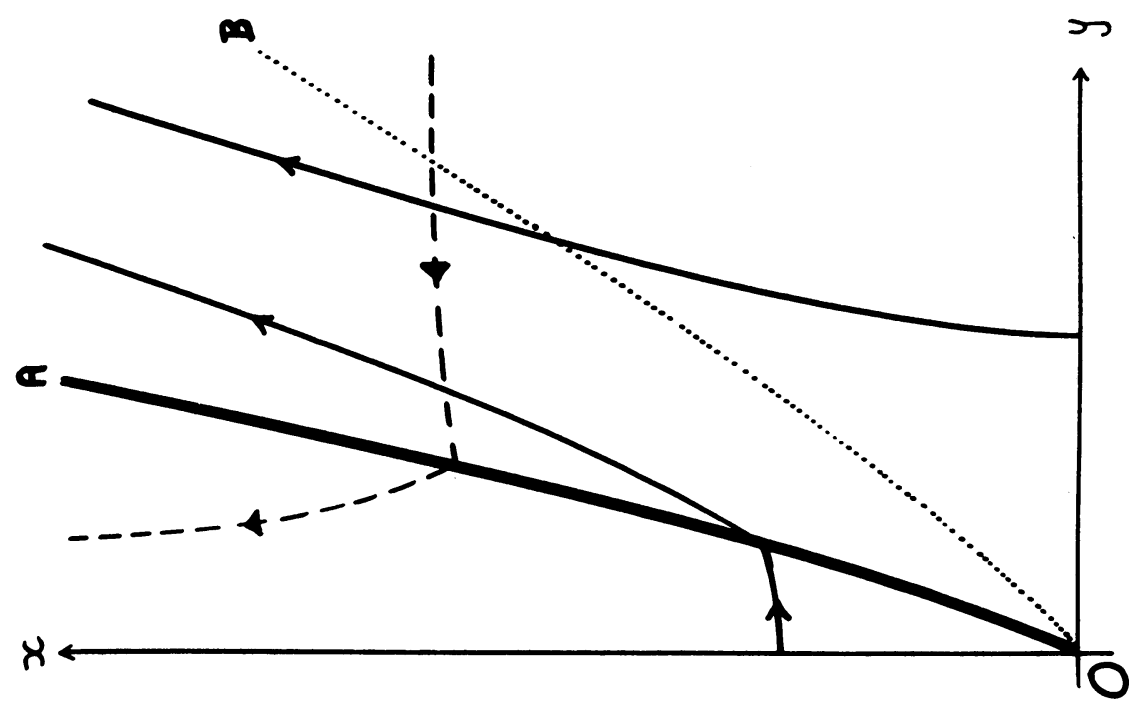

ำ

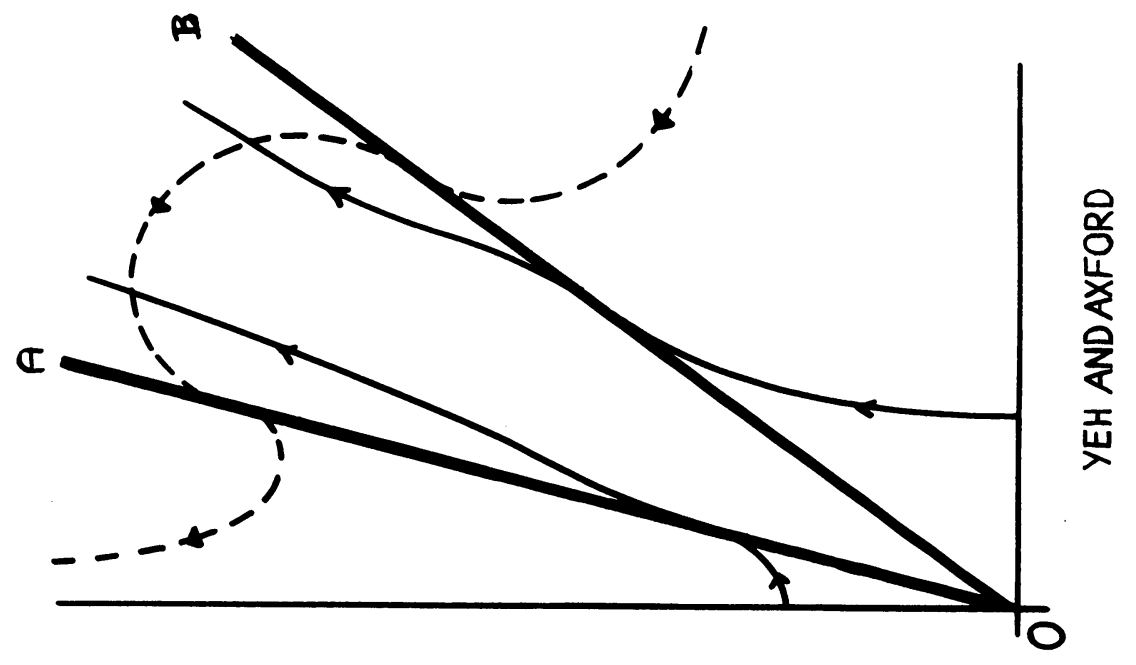




\subsection{SONNERUP}

The configuration proposed by Sonnerup (1970) is shown in Figure $2 b$. There are now two discontinuities in each quadrant. They are, however, different in nature. In the compressible case, $O A$ becomes a slow magnetohydrodynamic shock-wave, but $O B$ becomes an expansion fan. Furthermore, the wave $O A$ is generated at $O$, whereas $O B$ is generated at the corner $B$, a fact which makes the model inapplicable in detail to astrophysical problems. In spite of this fault, the model proves to be most useful because of its simplicity, which arises from the fact that the plasma velocity and magnetic field are both uniform in each of the three regions bounded by the $x$ - and $y$-axes, $O A$ and $O B$. The conservation relations across $O A$ and $O B$ thus enable one to relate the values in the three regions analytically.

The elegance of the model has made it easy for Cowley $(1974 a, b)$ to generalize it in two ways. Firstly, he includes magnetic field and plasma velocity components normal to the plane of Figure $2 b$ and finds that it is possible to choose the inflow and outflow values of the normal components arbitrarily and independently. Secondly, he is able to construct solutions in which the two inflowing magnetic fields differ in magnitude; for a given set of inflow parameters the solution is unique (Priest and Cowley, 1975). The generalization to include compressibility has not, however, been performed in a self consistent way; the most that has been done so far is a partially consistent treatment due to Yeh and Dryer (1973).

It may be argued that the wave $O B$ represents a lumping together for mathematical convenience of the magnetohydrodynamic interaction which exists to the right of $O A$ in Petschek's mechanism. But, in view of the fact that Sonnerup's model appears to yield no upper limit on the reconnection rate, by contrast with Petscheks model, such a representation is unlikely to be exact. It is therefore of importance to analyse Petschek's mechanism in more detail.

\subsection{YeH AND AXFord}

Yeh and Axford (1970) adopted a different philosophy to that of Petschek for seeking the external region flow. They argue that, on some scale length intermediate between the size of the diffusion region and the distance between the magnetic field sources, there is no natural scale length and the magnetohydrodynamic variables are of self-similar form. They express the plasma velocity $\mathbf{v}$ and magnetic induction $\mathbf{B}$ in terms of a stream function $\psi$ and vector potential $\boldsymbol{A}$ defined by

$$
\begin{gathered}
\left(v_{r}, v_{\theta}\right)=\left(\frac{1}{r} \frac{\partial \psi}{\partial \theta},-\frac{\partial \psi}{\partial r}\right), \\
\left(B_{r}, B_{\theta}\right)=\left(\frac{1}{r} \frac{\partial A}{\partial \theta},-\frac{\partial A}{\partial r}\right) .
\end{gathered}
$$

The equations to be solved for steady, two-dimensional, incompressible flow are

$$
\begin{aligned}
& \rho(\mathbf{v} \cdot \boldsymbol{\nabla}) \mathbf{v}=-\boldsymbol{\nabla}\left(p+B^{2} / 2 \mu\right)+(\mathbf{B} \cdot \boldsymbol{\nabla}) \mathbf{B} / \mu, \\
& \mathbf{E}+\mathbf{v} \wedge \mathbf{B}=0, \\
& \boldsymbol{\nabla} \cdot \mathbf{B}=\boldsymbol{\nabla} \cdot \mathbf{v}=0,
\end{aligned}
$$


where $\rho$ and $E$ are constants. The form for the solution which Yeh and Axford assume is

$$
\psi=r g(\theta), \quad A=r f(\theta) ;
$$

in other words the components of $\mathbf{v}$ and $\mathbf{B}$ depend on $\theta$ alone. The resulting ordinary differential equations for $g(\theta)$ and $f(\theta)$ are solved subject to the boundary conditions

$$
\psi=\frac{\partial A}{\partial \theta}=0 \text { on } \theta=0, \pi / 2
$$

where, as indicated in Figures $2 \mathrm{a}$ and 3 , we take for convenience the $x$-axis as vertical and measure $\theta$ from it. The conditions (3) arise from the symmetry assumptions that a streamline is traced by the $x$ - and $y$-axes while the magnetic field lines intersect it normally.

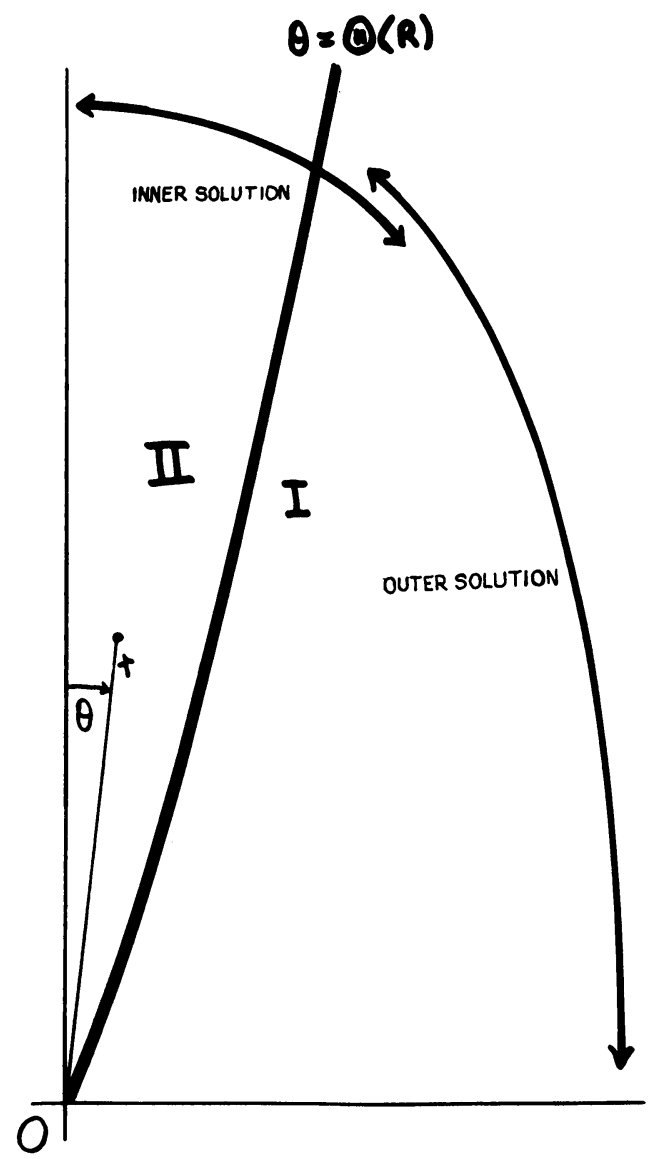

Fig. 3. Different domains for the solution. The discontinuity $O A$ (finite Alfvén wave) is situated at $\theta=\Theta(R)$, where $R=\log _{e}(r / l)+\pi /\left(8 M_{i}\right)$; it separates the first quadrant into regions I and II. The outer solution is valid from $\theta=\pi / 2$ down to small values of $\theta$, whereas the inner solution applies for $\theta / \Theta$ between zero and some large value. 
The resulting form for the streamlines and field lines is shown in Figure 2c. They are curved, except in the special case of a uniform total pressure (gas plus magnetic), when the model reduces to that of Sonnerup. As in Sonnerup's model, there are two straight discontinuities and again the wave $O B$ is subject to the criticism of being externally generated. More unluckily, however, the model does not work at all, because a detailed investigation of the nature of $O B$ (Vasyliunas, 1975) shows it to be unphysical; it is not possible to construct a solution which joins the flow on both sides except in the special case of Sonnerup's solution. The concept of looking for similarity solutions such as (2) is, however, important.

\section{An Asymptotic Similarity Solution for Fast Reconnection}

For reasons given in the previous section, Petschek's mechanism is the only fast reconnection model directly relevant to an astrophysical application. But a lingering doubt about it remains, because of both the ad-hoc way in which it was constructed and the semi-quantitative nature of Petschek's analysis. For these reasons, Soward and Priest (1976) have looked for two-dimensional solutions of the steady, incompressible magnetohydrodynamic equations (1) which are asymptotic in form and therefore valid at large distances from the diffusion region in the same spirit as Yeh and Axford's analysis. (The solutions, it transpires, have the added bonus of remaining valid right up to the diffusion region provided that the inflow Alfvén Mach number $M_{i} \equiv v_{i}(\mu \rho)^{1 / 2} / B_{i}$ is much less than unity.) We have asked the question "Just what is the distant external region flow for fast reconnection in practice with no externally generated discontinuities $O B$ present?" Is it qualitatively like Petschek's picture or is there, as suggested by Coppi and Friedland (1971), no need for any discontinuities at all?

There are two curves $O A, O B$ in each quadrant passing through the origin $O$ (Figure 2d) along which the normal components of plasma and Alfvén velocity are the same in magnitude. It does indeed prove possible to construct solutions which are continuous across $O B$, but across $O A$ the tangential components are forced to suffer discontinuities.

We first of all look for similarity solutions more general than (2), namely of the form

$$
\psi=r^{1-n} g(\theta), \quad A=r^{1+n} f(\theta),
$$

where $n>0$, so that the inertia forces are negligible as $r \rightarrow \infty$. But due to the singular behaviour of $\psi$ and $A$ at $O B$ we find similar difficulties at $O B$ to those unearthed by Vasyliunas in Yeh and Axford's case. The difficulties, however, become less severe in the limit as $n$ approaches zero. This leads us to search instead for solutions close to (2) in form but containing a weak (logarithmic) dependence on $r$. Specifically, we put

$$
\psi=r g(R, \theta), \quad A=r f(R, \theta),
$$

where

$$
R=\log _{e}(r / L)+R_{0}
$$


and $R_{0}$ is a constant, chosen for convenience (to make $B=B_{i}$ when $r=l$ ) as $\pi /\left(8 M_{i}\right)-\log _{e} M_{i}$.

Series solutions for $f$ and $g$ of the following form are then assumed:

$$
\begin{aligned}
& f=R^{1 / 2} f_{0}(\theta)+R^{-1 / 2}\left(f_{11}(\theta) \log _{e} R+f_{1}(\theta)\right)+\ldots, \\
& g=R^{-1 / 2} g_{0}(\theta)+R^{-3 / 2}\left(g_{11}(\theta) \log _{e} R+g_{1}(\theta)\right)+\ldots
\end{aligned}
$$

The largest powers of $R$ are determined to be $+\frac{1}{2}$ and $-\frac{1}{2}$ in order to make both the product $f g$ of order unity (see $1 b$ ) and the Lorentz force to dominate the inertial term in (1a). Having substituted into (1) we derive ordinary differential equations for the unknown functions of $\theta, f_{0}, f_{11}, f_{1}, g_{0}, g_{11}, g_{1}, \ldots$, by equating the coefficients of powers of $R$ in (1) to zero. The equations are then solved for $0<\theta \leq \pi / 2$ subject to the conditions

$$
g=\partial f / \partial \theta=0 \text { at } \theta=\pi / 2
$$

which result from (3). But the resulting solutions are found not to obey the remaining boundary conditions, namely

$$
g=\partial f / \partial \theta=0 \text { at } \theta=0 .
$$

The form (4) and (5) is therefore not valid right down to $\theta=0$; it represents only an 'outer' solution, as indicated diagrammatically in Figure 3.

The 'inner' solution, which holds for small values of $\theta$, is constructed by changing the variable from $\theta$ to

$$
\xi=\theta / \Theta(R),
$$

where

$$
\Theta(R)=\Theta_{o} R^{-1}+\left(\Theta_{11} \log _{e} R+\Theta_{1}\right) R^{-2}+\ldots
$$

is, at this stage, just a typical small value of $\theta$. We write as the inner solution

$$
\psi=r G(R, \xi), \quad A=r F(R, \xi),
$$

where

$$
F=R^{-1 / 2} F_{0}(\xi)+R^{-3 / 2}\left(F_{11}(\xi) \log _{e} R+F_{1}(\xi)\right)+\ldots
$$

and

$$
\left.G=R^{-1 / 2} G_{0}(\xi)+R^{-3 / 2}\left(G_{11}(\xi) \log _{e} R+G_{1}(\xi)\right)+\ldots .\right\}
$$

Again, having substituted for $\psi$ and $A$ in (1) and equated the coefficients of powers of $R$, we find a series of ordinary differential equations in $\xi$ for the unknown functions $F_{o}, F_{11}, F_{1}, G_{o}, G_{11}, G_{1} \ldots$ They are solved for $0 \leq \xi<\infty$, subject to the boundary conditions

$$
G=\partial F / \partial \xi=0 \quad \text { at } \quad \xi=0 .
$$


Finally, certain free parameters in the inner and outer asymptotic expansions (6) and (8) are determined after 'matching' the expansions by means of the conditions

$$
\lim _{\theta \rightarrow 0} g=\lim _{\xi \rightarrow \infty} G
$$

and

$$
\lim _{\theta \rightarrow 0} f=\lim _{\xi \rightarrow \infty} f
$$

to all orders.

The details of the above procedure for determining the inner and outer expansions are in practice far from simple and are given by Soward and Priest (1976). To lowest order we find Equation (1) for the outer region becomes

$$
\begin{aligned}
& \frac{\mathrm{d}^{2} f_{o}}{\mathrm{~d} \theta^{2}}+f_{o}=0, \\
& f_{o} \frac{\mathrm{d} g_{o}}{\mathrm{~d} \theta}-g_{o} \frac{\mathrm{d} f_{o}}{\mathrm{~d} \theta}=1,
\end{aligned}
$$

with solution, subject to the boundary conditions (7),

$$
f_{o}=-a \sin \theta, \quad g_{o}=a^{-1} \cos \theta,
$$

where $a$ is a constant.

In the inner region, on the other hand, (1) reduces to

$$
\begin{aligned}
& \left(G_{0}^{2}-F_{0}^{2}\right) \frac{\mathrm{d}^{2} G_{o}}{\mathrm{~d} \xi^{2}}=0, \\
& F_{o} \frac{\mathrm{d} G_{o}}{\mathrm{~d} \xi}-G_{o} \frac{\mathrm{d} F_{o}}{\mathrm{~d} \xi}=\Theta_{o},
\end{aligned}
$$

which are to be solved for $\xi \geq 0$ subject to the boundary conditions

$$
G_{o}=\mathrm{d} F_{o} / \mathrm{d} \xi=0 \text { at } \xi=0,
$$

resulting from (9). Near $\xi=0$, the solution of (12) is

$$
G_{o}=\text { const } \times \xi
$$

but it cannot hold for all values of $\xi$ since it is not possible to match it to the solution (11) in the sense of (10). It can be seen that the most general continuous solution of (12) is a linear function of $\xi$ with changes in gradient at the places where $G_{o}=F_{o}$ and $G_{o}=-F_{o}$, which correspond to the Alfvén curves $O A$ and $O B$, respectively. Without loss of generality, we assume the curve $\xi=1$ to be situated where $G_{o}=F_{o}$, so that, as indicated in Figure 3,OA is given by $\theta=\Theta(R)$. Furthermore, we suppose that $F_{o}$ and $G_{o}$ do suffer changes in gradient at $\xi=1$ but not at the curve where $G_{o}=-F_{o}$, since a discontinuity at $O A$ is generated from $O$ and so acceptable but one at $O B$ is generated from some external point $B$ and so is not acceptable on physical grounds. The resulting lowest order inner region solution of (12) and (13), 
which is capable of being matched to (11), is then

$$
\begin{array}{lll}
F_{o}=\sqrt{ } \Theta_{o}, & G_{o}=\sqrt{ } \Theta_{o} \xi & \text { in } \quad \text { II }(0 \leq \xi<1), \\
F_{o}=\sqrt{ } \Theta_{o}(2-\xi), & G_{o}=\sqrt{ } \Theta_{o} & \text { in } \quad \text { I }(\xi>1) .
\end{array}
$$

Finally, the matching condition (10) implies that

$$
a=\Theta_{o}^{-1 / 2} \text {. }
$$

The next order terms in the asymptotic expansions (6) and (8) are found in a straightforward manner and the matching condition leads to a value for $\Theta_{o}$ of $\pi / 8$, so that to lowest order the discontinuity $O A$ is located at

$$
\theta=\Theta \equiv \pi /(8 R) \text {. }
$$

At this order, however, a weak singularity in the behaviour of $F_{1}(\xi)$ and $G_{1}(\xi)$ near $\xi=1$ appears. In order to find a continuous transition through $O A$, it proves necessary to determine the inner and outer expansions to the next highest order (to terms of order $R^{-5 / 2}$, for instance, in $F$ and $G$ ) and to take account of finite conductivity effects near $O A$. The reader is referred to Soward and Priest (1976) for the details. It suffices here to state that having written $B^{\prime}=B B_{i}^{-1} M_{i}^{-1 / 2}, v^{\prime}=$ $v v_{i}^{-1} M_{i}^{1 / 2}$ we find that the lowest order contributions to each of the components of plasma velocity and magnetic induction are

$$
\left.\begin{array}{l}
B_{x}^{\prime}=-(8 R / \pi)^{1 / 2}, \quad B_{y}^{\prime}=(\pi R / 2)^{-1 / 2}(\pi / 2-\theta), \\
v_{x}^{\prime}=-(\pi / 32)^{1 / 2} R^{-3 / 2}(\pi / 2-\theta), \quad v_{y}^{\prime}=-(8 R / \pi)^{-1 / 2}
\end{array}\right\} \text { in I }
$$

and

$$
\left.\begin{array}{rl}
B_{x}^{\prime}= & -(\pi R / 2)^{-1 / 2} \log _{e}[(1+\xi) /(1-\xi)], B_{y}^{\prime}=-(8 R / \pi)^{-1 / 2}, \\
v_{x}^{\prime}= & (8 R / \pi)^{1 / 2}, \quad v_{y}^{\prime}=-(\pi / 32)^{1 / 2} \\
& \times \mathrm{R}^{-3 / 2}\left\{\log _{e}[(1+\xi) /(1-\xi)]-\xi\right\} .
\end{array}\right\} \text { in II }
$$

The dominant components are thus $B_{x}, v_{y}$ in I and $v_{x}$ in II. The form for the resulting field lines and streamlines is shown in Figure $2 \mathrm{~d}$, where the curve $O B$, through which there is a continuous transition, is shown dotted. The position of $O B$ has been exaggerated; both $O B$ and $O A$ are in fact situated close to $O x$ in the inner region. Also it should be noted that the curvature of the magnetic field lines in II allays the fears of Green and Sweet (1966) and Priest (1972) that Petschek's mechanism would not work.

Now, from (14), the inflow speed on the $y$-axis a distance $L_{e}$ from the origin is

$$
v_{e}=v_{i}\left(8 R_{e} M_{i} / \pi\right)^{-1 / 2}
$$

where, according to (5), the value of $R$ at $r=L_{e}$ is

$$
R_{e}=\pi /\left(8 M_{i}\right)+\log _{e}\left[L_{e} /\left(L M_{i}\right)\right] \text {. }
$$

Further, the inflow and outflow speeds $v_{i}$ and $v_{o}$, indicated on Figure 1, are related to $v_{e} b y$

$$
v_{i} B_{i}=v_{o} B_{o}=v_{e} B_{e}
$$


due to the constancy of the electric field. Equations (i), (ii), (iii), (15), (16), (17) may therefore be combined to yield the following relation between the Alfvén Mach numbers $M_{i} \equiv v_{i}(\mu \rho)^{1 / 2} / B_{i}$ and $M_{e} \equiv v_{e}(\mu \rho)^{1 / 2} / B_{e}$ on the $y$-axes at distances $l$ and $L_{e}$, respectively, from the neutral point:

$$
\frac{\pi}{8 M_{e}}=\frac{\pi}{8 M_{i}}+\frac{1}{2} \log _{e}\left(R_{m e}^{2} M_{i} M_{e}\right)
$$

where $R_{m e}=L_{e} v_{A e} / \eta$ is the magnetic Reynolds number based on the external conditions and $v_{\mathrm{A} e} \equiv B_{e}(\mu \rho)^{-1 / 2}$ is the Alfvén speed. Equation (18) is strictly valid only where $M_{i} \ll 1$, since, otherwise, the external region solution does not hold right down to the diffusion region. Nevertheless, we have sketched the solution in Figure 4

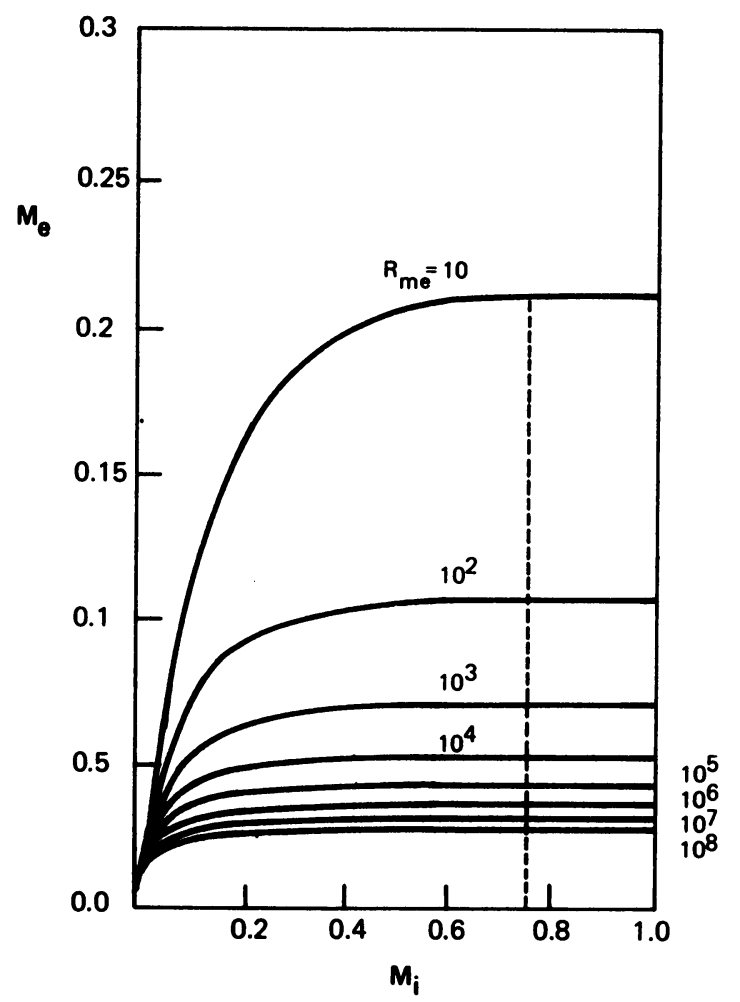

Fig. 4. The external Alfvén Mach number $M_{e}$ is plotted against the inflow Alfvén Mach number $M_{i}$ for various values of the magnetic Reynolds number $R_{m e}=v_{A_{e}} L_{e} / \eta$. The maximum values of $M_{e}$, namely $M_{e \max }$, are located at the intersection of the curves with the broken line.

for values of $M_{i}$ right up to unity. $M_{e}$, plotted as a function of $M_{i}$ alone, possesses a maximum value $M_{e \max }$, which occurs at $M_{i}=\pi / 4$, and which is sketched as a function of the magnetic Reynolds number in Figure $5 . M_{e \max }$ decreases from 0.2 , when $R_{m e}=10$ to 0.02 when $R_{m e}=10^{6}$ and, as can be seen from the figure, is somewhat less than the value derived from Petschek's original analysis. 


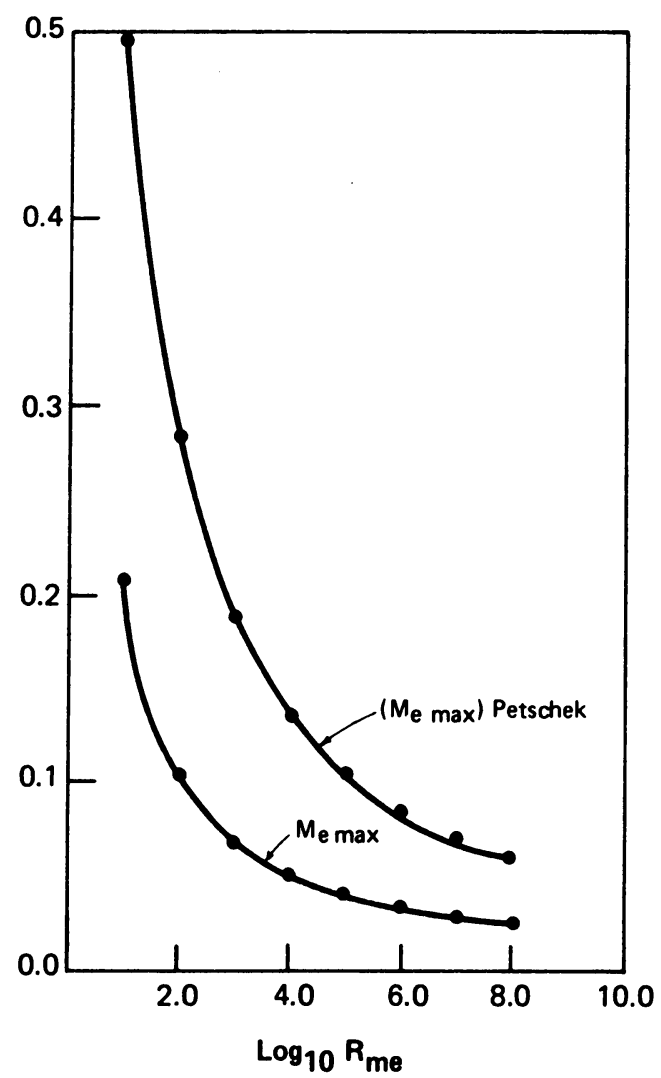

Fig. 5. $M_{e \max }$ from our analysis as a function of $\boldsymbol{R}_{m e}$. For comparison the corresponding value from Petschek's simpler analysis is also shown.

In conclusion, Petschek's mechanism has been shown to be the only workable model for fast reconnection in practice. It now has a sound mathematical basis. Reconnection can occur at any rate up to typically a tenth or a hundredth of the Alfvén speed, depending on the magnetic Reynolds number. By contrast with Petschek's original analysis, the shocks, magnetic field lines and streamlines are all in general curved in the manner indicated by Figure $2 \mathrm{~d}$.

\section{References}

Coppi, B. and Friedland, A. B.: 1971, Astrophys. J. 169, 379.

Cowley, S. W. H.: 1974a, J. Plasma Phys. 12, 319.

Cowley, S. W. H.: 1974b, J. Plasma Phys. 12, 341.

Dungey, J. W.: 1953, Phil. Mag. 44, 725.

Green, R. M. and Sweet, P. A.: 1966, Astrophys. J. 147, 1153.

Parker, E. N.: 1963, Astrophys. J. Suppl. Ser. 77, 8, 177.

Petschek, H. E.: 1964, AAS-NASA Symposium on the Physics of Solar Flares (ed. W. N. Hess), NASA SP-50, p. 425. 
Priest, E. R.: 1972, Monthly Notices Roy. Astron. Soc. 159, 389.

Priest, E. R. and Cowley, S. W. H.: 1975, J. Plasma Phys. 14, 271.

Roberts, B. and Priest, E. R.: 1975, J. Plasma Phys. 14, 417.

Sonnerup, B. U. O.: 1970, J. Plasma Phys. 4, 161.

Soward, A. M. and Priest, E. R.: 1976, submitted for publication.

Sweet, P. A.: 1958, Nuovo Cimento Suppl. 8, Ser. X, 188.

Vasyliunas, V. M.: 1975, Rev. Geophys. Space Phys. 13, 303.

Yeh, T. and Axford, W. I.: 1970, J. Plasma Phys. 4, 207.

Yeh, T. and Dryer, M.: 1973, Astrophys. J. 182, 301.

\section{DISCUSSION}

Gilman: How is your magnetic Reynolds number defined?

Priest: $R_{m e}=v_{\mathrm{Ae}} L_{e} \mu_{0} \sigma$ (see text), but one could just as easily express the results in terms of a magnetic Reynolds number based on $v_{e}$ rather than $v_{\mathrm{A} e}$.

Smith: It seems to me that your results will be applicable to crude models of a solar flare only if the conductivity $\sigma$ is taken as a turbulent conductivity so that $R_{m}$ is reduced from $10^{6}$, a number typical of the nonturbulent corona.

Priest: The reconnection model works whether you take a Coulomb or a turbulent value for $\sigma$. For a flare, there is probably an initial stage with the Coulomb value, which is then triggered to a main phase with the turbulent value. In each case (and for any astrophysical application), one needs to determine whether some additional consideration gives rise to an upper limit on $M_{e}$ which is less than $M_{e \max }$. For instance, one may require that the width $l$ of the diffusion region be greater than a collision mean-free path or an ion gyroradius. Whether this is a stringent limitation or not in practice depends crucially on how high in the solar atmosphere the reconnection is taking place. 